\title{
An extracytoplasmic function sigma factor-dependent periplasmic glutathione peroxidase is involved in oxidative stress response of Shewanella oneidensis
}

Jingcheng Dai ${ }^{1,2}$, Hehong Wei ${ }^{1,2}$, Chunyuan Tian ${ }^{3}$, Fredrick Heath Damron ${ }^{4}$, Jizhong Zhou ${ }^{{ }^{*}}$ and Dongru Qiu ${ }^{1,2^{*}}$

\begin{abstract}
Background: Bacteria use alternative sigma factors (ఠs) to regulate condition-specific gene expression for survival and Shewanella harbors multiple ECF (extracytoplasmic function) $\sigma$ genes and cognate anti-sigma factor genes. Here we comparatively analyzed two of the rpoE-like operons in the strain MR-1: rpoE-rseA-rseB-rseC and rpoE2-chrR.

Results: RpoE was important for bacterial growth at low and high temperatures, in the minimal medium, and high salinity. The degP/htrA orthologue, required for growth of Escherichia coli and Pseudomonas aeruginosa at high temperature, is absent in Shewanella, while the degQ gene is RpoE-regulated and is required for bacterial growth at high temperature. RpoE2 was essential for the optimal growth in oxidative stress conditions because the rpoE2 mutant was sensitive to hydrogen peroxide and paraquat. The operon encoding a ferrochelatase paralogue $(\mathrm{HemH} 2)$ and a periplasmic glutathione peroxidase (PgpD) was identified as RpoE2-dependent. PgpD exhibited higher activities and played a more important role in the oxidative stress responses than the cytoplasmic glutathione peroxidase CgpD under tested conditions. The rpoE2-chrR operon and the identified regulon genes, including pgpD and hemH2, are coincidently absent in several psychrophilic and/or deep-sea Shewanella strains.

Conclusion: In S. oneidensis MR-1, the RpoE-dependent degQ gene is required for optimal growth under high temperature. The rpoE2 and RpoE2-dependent pgpD gene encoding a periplasmic glutathione peroxidase are involved in oxidative stress responses. But rpoE2 is not required for bacterial growth at low temperature and it even affected bacterial growth under salt stress, indicating that there is a tradeoff between the salt resistance and RpoE2-mediated oxidative stress responses.
\end{abstract}

Keywords: Periplasmic glutathione peroxidase, Shewanella, ECF sigma factor, Oxidative stress response

\section{Background}

The $\gamma$-proteobacteria Shewanella species have two hallmark traits, respiratory versatility and psychrophily $[1,2]$. Respiratory versatility is characterized by their ability to utilize a series of organic and inorganic electron acceptors, particularly metals and metalloids of $\mathrm{Fe}(\mathrm{III}), \mathrm{Mn}(\mathrm{IV}), \mathrm{Ur}(\mathrm{VI})$ and the direct electron transfer

\footnotetext{
* Correspondence: jzhou@ou.edu; qiu@ihb.ac.cn

5 Institute for Environmental Genomics and Department of Botany and Microbiology, The University of Oklahoma, Stephenson Research and Technology Center, 101 David L. Boren Blvd, Norman OK 73019, USA 'Institute of Hydrobiology, Chinese Academy of Sciences, 7 South Donghu Road, Wuchang District, Wuhan 430072, China

Full list of author information is available at the end of the article
}

to electrodes [3,4]. Shewanella species harbor a variety of outer membrane and periplasmic $c$-type cytochrome genes expressed for respiration under different environmental conditions. Bacterial gene expression is regulated by a series of transcriptional factors including alternative sigma factors $\left(\sigma^{\mathrm{S}}\right)$. Sigma factors are a component of bacterial RNA polymerase (RNAP) and determine promoter selectivity of the holoenzyme, thus playing a central role in the regulation of gene expression. Bacteria usually have one housekeeping $\sigma$ factor (RpoD) and a variable number of alternative $\sigma$ factors that possess different promoter-recognition properties [5]. The number of alternative $\sigma$ factors highly varies among bacteria and may 
be related to their specific habitat, metabolisms, and development [5-9]. Extracytoplasmic function (ECF) $\sigma$ factors are highly regulated factors that control expression of genes and constitute the third pillar of bacterial signal transduction after the one-component and two-component systems [9]. Most ECF os are sequestered by an anti-sigma factor, which can be deactivated by proteolysis, conformational change, partner switching (including mimicry) or other unknown mechanisms to release the ECF sigma factor from being sequestered [9]. Once the ECF sigma factor is released it can then activate of transcription of regulon genes throughout the genome. ECF sigma factor RpoE and its regulators have been extensively studied in $E$. coli [10-17], Pseudomonas aeruginosa [18-22] and Bacillus subtilis [6,7]. RpoE regulates a series of extracytoplasmic functions, including synthesis of envelope proteins, outer membrane protein (OMP) modification, cell envelope structure and cell division in E. coli [23]. The RpoE counterpart $\mathrm{AlgU} / \mathrm{T}$ controls the production of a series of pathogenic factors, lipoproteins, and the extracellular polysaccharide alginate in $P$. aeruginosa which causes the mortality and morbidity of patients with cystic fibrosis [24-26].

The sigma factors of Shewanella have remained relatively uncharacterized. The genome of Shewanella oneidensis MR-1 encodes 10 sigma factors (RpoD, RpoH, RpoS, RpoN, FliA, and five ECF sigma factors RpoE, RpoE2, SO_3551 (ECF-like), SO_3096 (ECF-like) and SO_3840 (ECF-like). Sigma32 $(\mathrm{RpoH})$ is the heat shock response sigma factor and it has been shown that heat shock activates expression of 323 genes and represses expression of 286 genes $[27,28]$. In $S$. violacea strain DSS12, three RpoE-like sigma factors have been identified [29,30]. Numerous transcriptomic studies have shown Shewanella can modulate gene expression in response to its environmental signals [29-37]. To shed light on the role of two of the RpoE sigma factors of $S$. oneidensis MR-1, comparative studies were conducted in this study. Deletion mutants were generated and utilized to ascertain the specific functions of each RpoE sigma factor and the two sigma factors dependent genes were identified. RpoE was required for growth at cold and high temperatures, in minimal media, and in high salt environments. Unlike RpoE, RpoE2 is responsible for resistance to oxidative stress. PgpD was identified as the RpoE2 dependent periplasmic glutathione peroxidase that facilitates resistance to oxidative stress. Understanding the regulation of RpoE and RpoE2 and the genes they control can help explain the ability of $S$. oneidensis to survive against environmental stress.

\section{Results}

RpoE ECF sigma factors and anti-sigma factor genes in

\section{S. oneidensis MR-1}

The homologues for the E. coli primary $\sigma$ factor, RpoD, and five out of six alternative $\sigma$ factors RpoN, RpoS, $\mathrm{RpoH}, \mathrm{RpoE}$, and FliA (RpoF), are present in all the sequenced genomes of Shewanella (data not shown). Several Shewanella strains such as S. baltica OS155 and S. putrefaciens W3-18-1 also contain another FliA for lateral flagella [38]. However, the FecR (anti-sigma factor)-FecI (sigma factor)-FecA (ferric citrate receptor) iron-starvation signaling system is absent in most of the sequenced Shewanella strains, except for a few S. baltica strains. There are five ECF-like $\sigma$ factors, encoded by SO_1342, SO_1986 SO_3096, SO_3551, and SO_3840, found in S. oneidensis MR-1. SO_1342 was identified as the orthologue for $\operatorname{rpoE}\left(\sigma^{\mathrm{E}}\right)$ of $E$. coli and algU/T of $P$. aeruginosa based on the high sequence similarity and the well-conserved gene cluster of rpoE-rseA-rseB-rseC and flanking genes (Figure 1). SO_1986 (designated rpoE2 hereafter) encodes the orthologue for RpoE of the photosynthetic $\alpha$-proteobacterium Rhodobacter sphaeroides, and the downstream locus SO_1985 encodes the putative cognate anti- $\sigma$ factor homologous to ChrR [39]. We further characterized the cellular functions of $\operatorname{rpoE}\left(\sigma^{\mathrm{E}}\right)$ and rpoE2 experimentally and computationally.

\section{RpoE and RpoE2 of S. oneidensis are responsible for diverse stress responses}

In order to characterize the roles of each of the RpoE sigma factors, the rpoE and rpoE2 genes were deleted from strain MR-1. The rpoE and rpoE2 mutant strains had no observable growth defects in rich media (Figure 2A). We examined the role of the rpoE sigma factor genes in growth under the stress conditions. The rpoE mutant displayed a severe growth defect when cultured in the minimal medium, but no growth defect was observed for

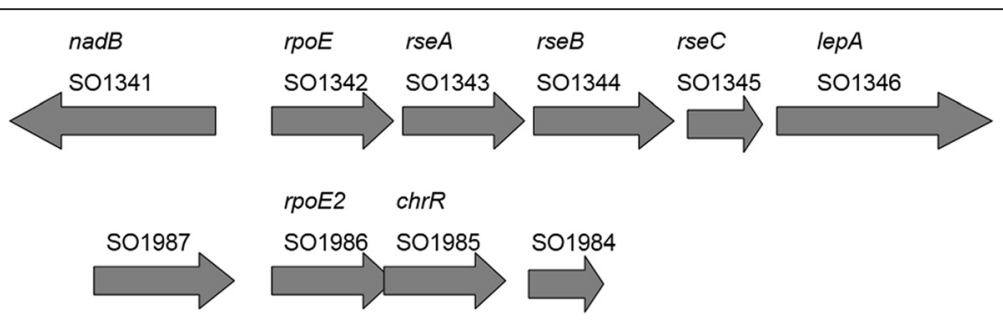

Figure 1 The gene clusters of $r p o E-r s e A-r s e B-r s e C$ and $r p o E 2-C h r R$ and the flanking loci on the chromosome of the S. oneidensis MR-1 strains. The conserved gene cluster rpoE-rseA-rseB-rseC and the flanking genes are also found in the genomes of Escherichia coli and Pseudomonas aeruginosa. 


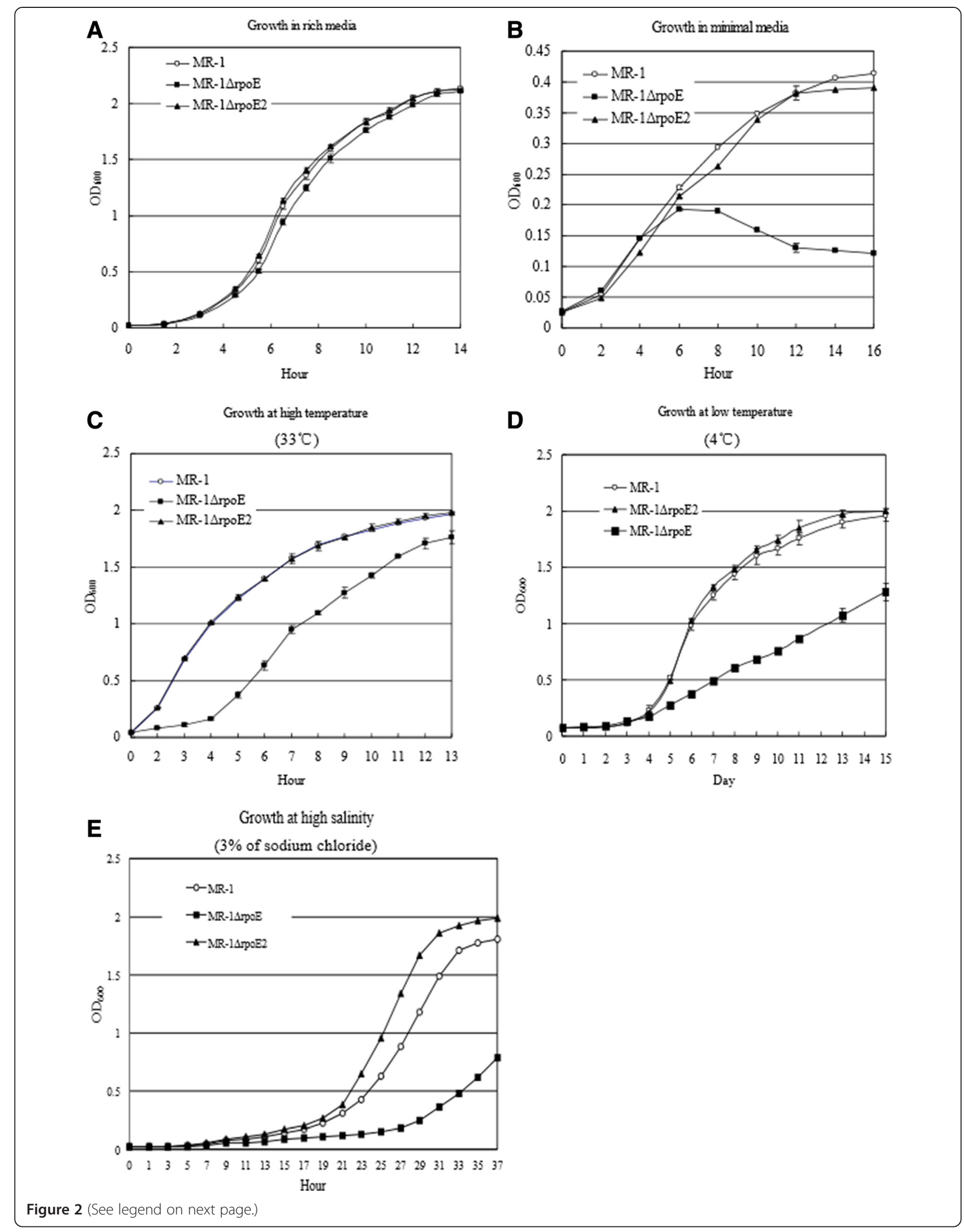


(See figure on previous page.)

Figure 2 The $r p o E$ mutant had growth defects when cultured in minimal media, high salinity, and high or low temperature. Bacterial growth, as measured by $\mathrm{OD}_{600}$, are show for the strains growing in various conditions: A) Rich medium (LB broth); B) Nutrient-poor environment (the modified M1 minimal medium); C) Higher temperature (at $33^{\circ} \mathrm{C}$ and in the LB medium), D) Low temperature (at $4^{\circ} \mathrm{C}$ and in the $L B$ medium); E) High salt stress (LB medium supplemented with $3 \%$ of sodium chloride, $w / v$ ).

the rpoE2 mutant strain (Figure 2B). As expected the rpoE mutant had a growth defect at high temperature $\left(33^{\circ} \mathrm{C}\right)$ however no growth defect was observed for the rpoE2 mutant (Figure 2C). In addition, the rpoE mutant also showed a growth defect at low temperatures $\left(4^{\circ} \mathrm{C}\right.$, Figure $2 \mathrm{D}$ and $10^{\circ} \mathrm{C}$, Additional file 1: Figure S1) and high salinity (LB medium supplemented with $3 \%$ of sodium chloride, w/v) (Figure 2E). Though the rpoE mutant showed an apparent growth defect at high salinity, the growth of the rpoE2 mutant was even better than that of the wild type strain (Figure $2 \mathrm{E}$ and Additional file 1: Figure S1). The rpoE mutant was susceptible to ampicillin (data not shown), though the S. oneidensis MR-1 wild type strain is resistant to this antibiotic [40].

\section{RpoE is autoreglated and DegQ is RpoE-dependent}

The multiple aligment and sequence logos analyses of promoter sequences upstream of rpoE/algU in Shewanella oneidensis, Pseudomonas aeruginosa and Escherichia coli were shown the conserved -35 and -10 motifs GAACTT--16/17 bp-TCCAAA upstream of rpoE/algU (Additional file 1: Figure S2). By using the Clustal W multiple alignment and Weblogo software[23], we also identified two conserved motifs GAACTT and TCTACA upstream of rpoE in 17 Shewanella strains, which are similar to the -35 and -10 consensus sequences of the RpoE-dependent promoter (Additional file 1: Table S3). Furthermor, we mapped the transcription start site (TSS) of rpoE (SO_1342) by using primer extension (Additional file 1: Figure S3A). In addition, expression of the pHERD30TrpoE in trans did significantly enhance the transcription of chromosomal $r s e A$ locus in the MR-1 strain, indicating that the expression of the rpoE-rseABC gene cluster could be up-regulated by RpoE (i.e., autoregulation, Figure 3).

Based on the promoter motif recognition and the knowledge on E. coli and P. aeruginosa, part of the RpoE-dependent regulon was predicted in the genome of MR-1 (Table 1). These genes encode the OMP assembly complex BamABCDE and the lipopolysaccharide (LPS) assembly complex components LptABCD and lipid biosynthesis-related proteins LpxA, B, and D. The $f k p A$, surA, skp and ppiA genes are involved in the proper folding of OMPs [17]. The expression of $\operatorname{degP} /$ $m u c D$ gene, encoding the periplasmic protease Do, is RpoE/AlgU-dependent and is required for survival at high temperatures and envelope integrity in E. coli and Pseudomonas because DegP/MucD could scavenge abnormal proteins in the periplasm and function as a chaperone for assembly of OMPs [23,24]. However, the only one DegP/HtrA homolog (encoded by SO_3942) was identified as the E. coli DegQ orthologue other than DegP because it lacks the characteristic Q-linker (residues 55-79) of the latter (Additional file 1: Figure S4) [41,42]. In addition, this gene $(\operatorname{deg} Q)$ is chromosomally linked with $\operatorname{degS}$ (SO_3943) as previously found in E. coli. The deletion of $\operatorname{deg} Q$ also resulted in the susceptibility of $S$. oneidensis MR-1 to ampicillin (data not shown). The $\operatorname{deg} Q$ gene does not belong to RpoE regulon in E. coli $[23,43]$ and is absent in Pseudomonas. We found that the $\operatorname{deg} Q$ gene was also RpoE-regulated in MR-1 because the induced expression of $r p o E$ enhanced the transcription of $\operatorname{deg} Q$ in turn (Figure 3). There is a TA rich region followed by the GAACTT motif upstream of the open reading frame of $\operatorname{deg} Q$ [27]. The heat shock sigma 32 factor gene $(\mathrm{rpoH})$ is also regulated by RpoE in MR-1 (Table 1). DegQ might act as a major protease for protein quality control in the periplasm in the absence of DegP. Deletion of $\operatorname{deg} Q$ resulted in severe growth defectiveness under a higher temperature and the growth of mutant could be rescued by genetic complementation of plasmid borne-degQ gene (Figure 4). These results showed that $\operatorname{deg} Q$ played a central role in the high temperature growth of Shewanella in the absence of the RpoE-dependent protease Do (DegP).

\section{RpoE2 mediates resistance to oxidative stress responses}

The rpoE2-chrR operon is present in S. oneidensis MR-1 (Figure 1 and Additional file 1: Figure S5, S6 and S7) and is absent in E. coli and Pseudomonas. The open reading frames (ORFs) of rpoE2 (SO_1986) and chrR (SO_1985) are overlapped and the overlapped sequence (ATGATTAA) contains the start codon (ATG) of chrR and the stop codon (TAA) of rpoE2, strongly suggesting that they belong to the same operon and are translationally coupled (Figure 1). The rpoE2 mutant was more sensitive to hydrogen peroxide and paraquat than the wild type MR-1 strain (Figure 5), indicating that RpoE2 is involved in the oxidative stress responses.

\section{Identification of the RpoE2 regulon of S. oneidensis}

Multiple alignment analyses on the nucleotide sequences upstream of rpoE2-chrR revealed two well-conserved motifs, TGATCC and CGTATT, similar to the -35 and -10 elements of RpoE-dependent promoter in $R$. sphaeroides 

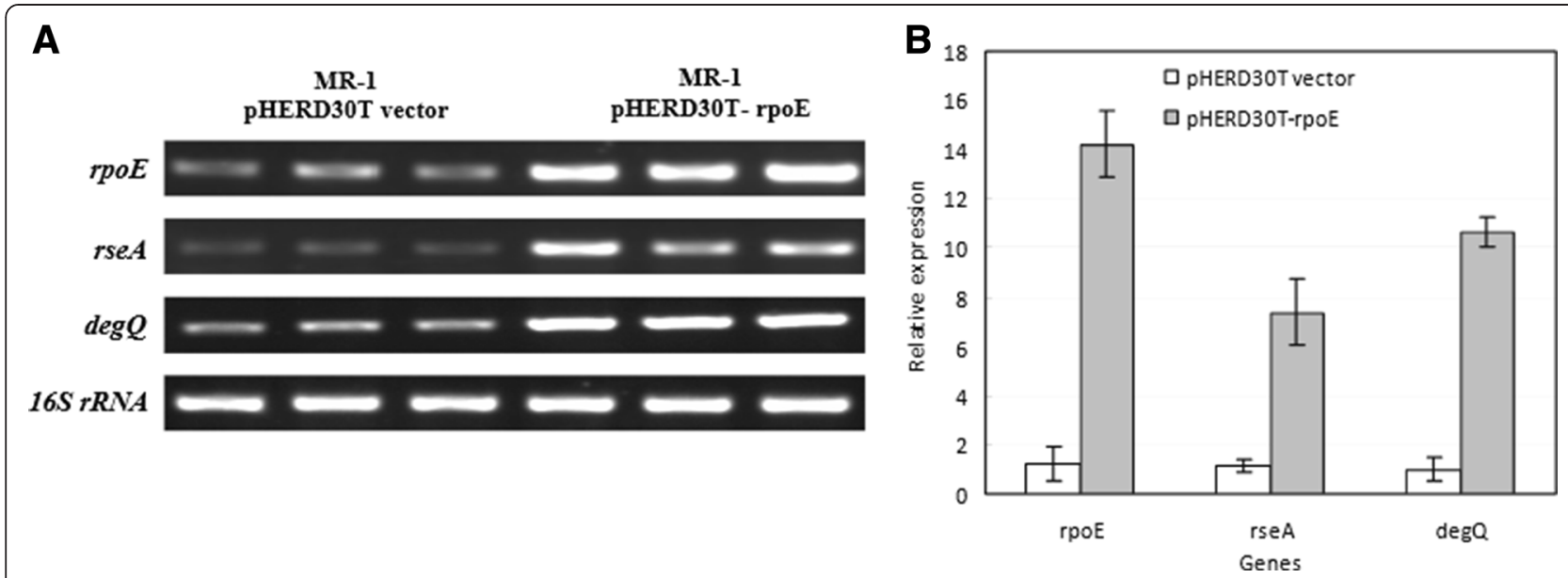

Figure 3 Expression of the $r p o E$ in the Shewanella oneidensis MR-1 activates expression of $r p o E$, rseA, and degQ. Strain MR-1 carrying the pHERD30T-rpoE plasmid was grown in the presense of L-arabinose $(0.05 \% \mathrm{w} / \mathrm{v})$. The MR-1 strain carrying only empty pHERD30T (with the pBAD promoter) vector was used as control. Note that the transcripts of $r p o E$ in the treatment (the right lanes) include the in trans expression of plasmid-borne rpoE gene, which further enhances the expression of chromosomal loci of rpoE (SO_1342), rseA (SO_1343), and degQ (SO_3942). The transcription of the chromosomal rpoE gene and down-stream cognate anti-sigma factor gene rseA is driven by the same promoter, and an RpoE-recognized promoter for autoregulation has been identified upstream of the rpoE-rseA-rseB-rseC operon. The cells were collected for RNA extraction after 1 hour of induction. A) Transcription of the genes was examined by using semi-quantitative RT-PCR; 16S rRNA gene expression was analyzed and used as the loading control. B) Trace quantity plotting of Figure 3A using 'Quantity One' software. The assays were performed in triplicates.

(Additional file 1: Figure S6). Furthermore, we mapped the transcription start site of rpoE2 (SO_1986) by using primer extension and RT-PCR methods (Additional file 1: Figure S3B and S7). The transcription of rpoE2 started from $\mathrm{A}(+1)$ downstream of the predicted -35 and -10 promoter motifs (Additional file 1: Figure S3B). The core regulon of RpoE2 had been previously predicted based on the promoter consensus sequence in the Vibrio-Shewanella species $[44,45]$, including $c f a$ (SO_3379, encoding cyclopropane fatty acid synthase) and phrB (SO_3384, deoxyribodipyrimidine photolyase). The loci SO_3379 (cfa) and SO_3384 (phrB) obviously belong to the same operon ranging from SO_3386 to SO_3374. By promoter recognition, we were also able to identify other candidates of RpoE2 regulon, including SO_3348 (encoding a ferrochelatase paralogue $\mathrm{HemH} 2$ homologous to $\mathrm{HemH}$ involved in heme biosynthesis), SO_3349 (a glutathione peroxidase located in the periplasm), SO_4169 (photolyase), SO_4170 (short chain dehydrogenase), and SO_1987 (Lon domain protease) (Additional file 1: Figure S8). These genes probably represent part of the core regulon of RpoE2 coping with photoreactive and oxidative stresses (Additional file 1: Figure S8 and Table 1). Our results also showed that the transcription of rpoE2 and chrR was induced by addition of hydrogen peroxide $(3 \mathrm{mM})$ (Additional file 1: Figure S9). We conducted the semi-quantitative RT-PCR analyses on the RpoE2-induced transcription of several genes of these operons/gene clusters (Figure 6). The L-arabinose induced expression of pHERD30T-borne rpoE2 remarkably increased the transcription of the chromosomal genes chrR,
SO_1987, SO_3349, SO_3386, and SO_4169 in the rpoE2 null mutant. These results indicate that the rpoE2-chrR pair is autoregulated and these genes belong to the RpoE2 regulon.

\section{RpoE2-dependent periplasmic hydrogen peroxidase is involved in oxidative stress response}

In light of the fact that RpoE2 plays a role in resistance to oxidative stress, we looked at the RpoE2 regulon for genes that encode proteins that could be responsible. Notably, the RpoE2 regulon member SO_3349 encodes a periplasmic gluthathione peroxidase D (designated $p g p D$ hereafter), which may be required for coping with the oxidative stress in the compartment of periplasm. The pgpD and the downstream hemH paralogue (SO_3348) had not been previously identified as the RpoE-ChrR regulon members in the photosynthetic $\alpha$-bacterium Rhodobacter sphaeroides. The PhoA-fusion assays [46] demonstrated that PgpD is secreted into the periplasm as previously predicted because the signal peptide of PgpD could mediate the secretion of PhoA (Additional file 1: Figure S10). We also mapped the transcription start site of the predicted RpoE2 regulon member SO_3349 and it is shown that the transcription of $p g p D$ (SO_3349) does start from the nucleotide A (+1) downstream of the -35 (TGATCC) and -10 (CGTAAT) promoter motifs as it was shown (Additional file 1: Figure S3C and S7). We have generated the in-frame deletion mutants of $p g p D$ and $\operatorname{cgpD}$ and tested the sensitivity of the mutants to hydrogen peroxide and paraquat. Our results showed that the $p g p D$ 
Table 1 Promoter motifs-based prediction of RpoE and RpoE2 regulon members in the genome of Shewanella oneidensis MR-1

\begin{tabular}{|c|c|c|c|c|}
\hline $\begin{array}{l}\text { Gene } \\
\text { identity }\end{array}$ & $\begin{array}{l}\text { Putative RpoE-dependent promoter } \\
\text { sequence }\end{array}$ & $\begin{array}{l}\text { Gene } \\
\text { name }\end{array}$ & Functions & Other genes in the operon \\
\hline SO_0516 & GAACTTATGTTTAAAATGACTGTCAGA & & Hypothetical protein & \\
\hline SO_1065 & GAACTTGCTCCTAAAGTTGGTGTCTCT & $f k p A$ & $\begin{array}{l}\text { FKBP-type peptidyl prolyl cis-trans } \\
\text { isomerase }\end{array}$ & \\
\hline SO_1342 & GAACTTITCAAAGTACGCGAGTCTAC & rpoE & RNA polymerase sigma 24 factor & $\begin{array}{l}\text { rseA(SO_1343)-rseB(SO_1344)-rseC } \\
\text { (SO_1345) }\end{array}$ \\
\hline SO_1476 & GAACTAAAACCCGCGGCTTAGGTCGAA & bamE & $\begin{array}{l}\text { Outer membrane protein (OMP) } \\
\text { assembly complex subunit } \mathrm{E}\end{array}$ & \\
\hline SO_1492 & GAACTTCTCTTCACACCTCGCСАСТАT & ppiA & Peptidyl prolyl cis-trans isomerase A & \\
\hline SO_1636 & GAACCTITAGATTITTTCAAAGTCGGA & rsep & $\begin{array}{l}\text { Membrane associated zinc } \\
\text { metalloprotease }\end{array}$ & $\begin{array}{l}\text { bamA(SO_1637)-skp(SO_1638)- IpxD } \\
\text { (SO_1639)-fabZ(SO_1640)- IpxA(SO_1641)- } \\
\text { IpxB(SO_1642)-rnhB(SO_1643) }\end{array}$ \\
\hline SO_1880 & GAACTTTCTGAGCAATGTCATGGTCTGT & bamc & OMP assembly complex subunit C & \\
\hline SO_3309 & GAACTCAAAGGCGACTTCTTTGTTCGT & bamB & OMP assembly complex subunit B & \\
\hline SO_3580 & GAACCGTACCCGCGTTTGGGGTCCAA & bamD & OMP assembly complex subunit D & SO_3581 \\
\hline SO_3636 & CAACTTTCCCCGTCGATACTTGTCCAG & IptD & $\begin{array}{l}\text { Lipopolysaccharide (LPS) transporter } \\
\text { subunit D }\end{array}$ & $\begin{array}{l}\text { surA(SO_3637)-pdxA(SO_3638)- } \\
\text { ksgA(SO_3639) }\end{array}$ \\
\hline SO_3637 & GAACCTCAACAAGGACTGAGAGTCCAA & surA & LPS assembly protein & pdxA(SO_3638)-ksgA(SO_3639) \\
\hline SO_3942 & GAACTTIITCAATGAGGTGCGTGTCCGA & $\operatorname{deg} Q$ & Periplasmic serine protease & \\
\hline SO_3958 & GAACTGCTATCGATCTACAATGTCACC & IptC & LPS transporter (LPT) subunit C & IptA(SO_3959)-IptB(SO_3960) \\
\hline SO_3959 & GAACTCGATCTCAACACTATGATAATG & IptA & LPS transporter subunit A & IptB(SO_3960) \\
\hline SO_4562 & GAACTTTAGCGTGTAAAATCACTCTATG & & Conserved hypothetical protein & \\
\hline \multirow[t]{2}{*}{ SO_4583 } & GAACTTITGTTCACTTGCAATGTCTAT & rpoH & RNA polymerase sigma 32 factor & \\
\hline & RpoE2-dependent promoter sequence & & & \\
\hline SO_1986 & TGATCCATTATTCAAAGGGCCACGTATT & rpoE2 & ECF RNA polymerase & $\operatorname{chrR}\left(S_{-}{ }_{1}\right.$ 1985, anti-sigma factor) \\
\hline SO_1987 & TGATCAAATTCTGATGATGGTACGTAAT & Lon & Lon domain protease & \\
\hline SO_3349 & TGATCCCTATCGTAGCAAGTTACGTAAT & $p g p D$ & Periplasmic glutathione peroxidase & hemH2 (SO_3348, ferrochelatase) \\
\hline SO_3386 & TGATCCITGTACAAGAATGGTCCGTAAT & $y b g A$ & $\begin{array}{l}\text { Photoreactivation-associated inner } \\
\text { membrane protein }\end{array}$ & $\begin{array}{l}\text { phrB (SO_3384, deoxyribo-dipyrimidine } \\
\text { photolyase) cfa (SO_3379, cyclopropane } \\
\text { fatty acid synthase) }\end{array}$ \\
\hline SO_4169 & TGATCCTCACAGTGCTGCTATCCGTAAC & phr & $\begin{array}{l}\text { Deoxyribodipyrimidine } \\
\text { photolyase-related protein }\end{array}$ & $\begin{array}{l}\text { SO_4170 (CsgA short chain } \\
\text { dehydrogenase/reductase) }\end{array}$ \\
\hline
\end{tabular}

deletion mutant (MR-1 $\Delta \mathrm{pgpD})$ exhibited a significantly higher sensitivity to oxidative stresses than the MR-1 strain $(p<0.01)$ while no remarkable difference was observed between the cgpD mutant $(\mathrm{MR}-1 \Delta \mathrm{cgpD})$ and wild-type strain under the tested concentrations (Figure 5). The growth defectiveness of the $p g p D$ deletion mutant in the presence of hydrogen peroxide and paraquat could be rescued by genetic complementation of plasmid borne-pgpD gene (Additional file 1: Figure S11). Though PgpD obviously plays a more important role than CgpD under our tested conditions, the double mutant $(\mathrm{MR}-1 \Delta \mathrm{cgpD} \Delta \mathrm{pgpD})$ was more sensitive to hydrogen peroxide stress than the MR-1 $\Delta$ rpoE2 and MR-1 $\Delta$ pgpD single mutants (Figure 5), indicating that the $c g p D$ gene is also involved in oxidative stress responses.
Expression and activity assays of cyptoplasmic and periplasmic hydrogen peroxidases

The cytoplasmic glutathione peroxidase $\mathrm{CgpD}$ and the periplasmic PgpD (residues 20-177) lacking the N-terminal signal peptide (MMKFPLFILTSLMSTSVFA) were successfully overproduced in the E. coli BL21/DE3 strain and were purified by Ni-NTP chromatography (Figure 7). Both CgpD and PgpD exhibited the hydrogen peroxide degradation activities in the presence of glutathione $(\mathrm{GSH})$ and the activity of PgpD was higher than that of CgpD under the conditions described (Figure 7). The glutathione export system genes are also present in the genome of MR-1, and are probably involved in the export of GSH from cytoplasm to periplasm. These results, together with the in vivo assays (Figure 5), strongly indicated that both PgpD and CgpD were 


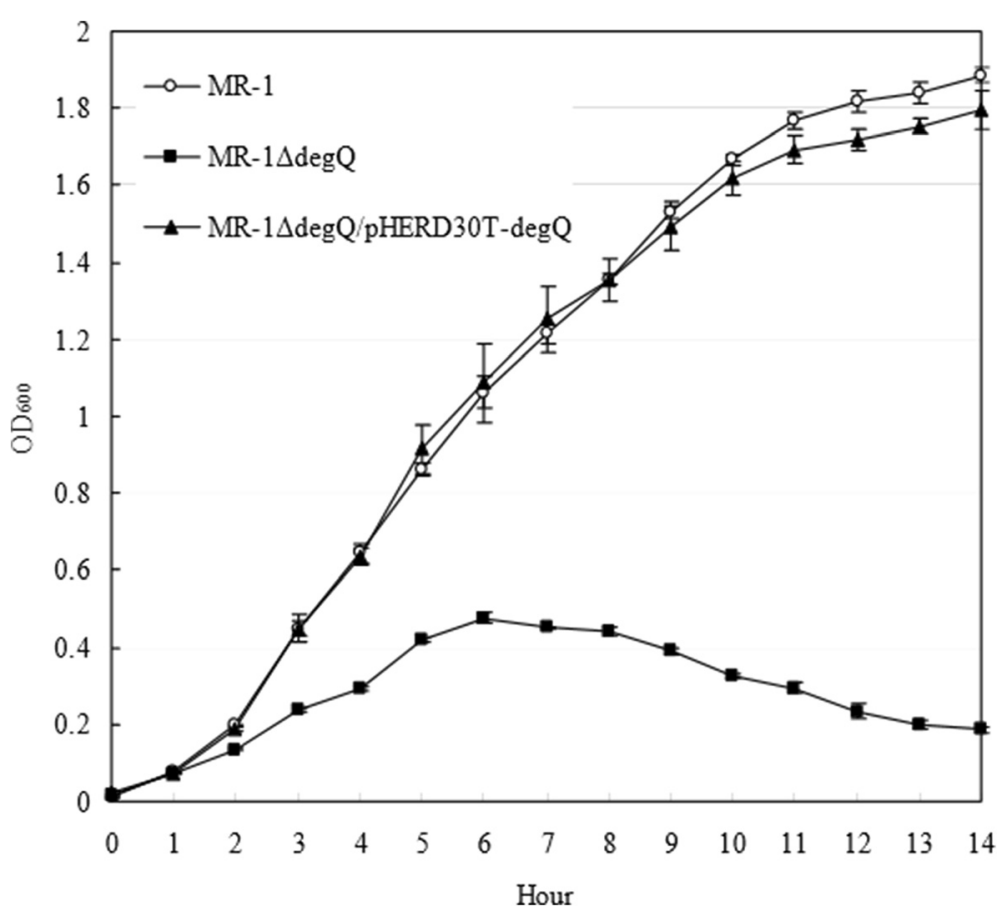

Figure 4 DegQ is required for optimal growth of strain MR-1 under high temperature. Genetic complementation by plasmid-borne degQ rescued bacterial growth of the degQ mutant. The MR-1 $\triangle$ degQ strains carrying empty vector (labeled as MR-1 $\triangle$ degQ) and pHERD30T-degQ plasmid (MR-1 $\triangle$ degQ+degQ) and the pHERD30T-carrying wild type MR-1 (MR-1) strains were grown in LB broth supplemented with $15 \mu \mathrm{g} / \mathrm{ml}$ of gentamycin. Bacterial strains were grown at $35^{\circ} \mathrm{C}$.

functional in Shewanella oneidensis and were probably involved in the degradation of hydrogen peroxide in the periplasm and cytoplasm compartments, respectively.

\section{Absence of RpoE2-ChrR pair and the regulon members in psychrophilic and/or deep-sea strains}

The RpoE2-ChrR system and the regulon members of RpoE2 may play a crucial part in coping with environmental stresses such as UVA radiation and more importantly reactive oxygen species (ROS) in Shewanella. The ROS could be sensed by ChrR, which undergoes conformational changes and releases the sequestered RpoE2. The released RpoE2 undergoes auto-upregulation by binding to the promoter of rpoE2-chrR operon and then drives the expression of enzymes involved in modification of cell membrane (Cfa), DNA damage repair ( $\mathrm{PhrB})$, degradation of ROS (PgpD) and other stress responses. Our comparative genomic analysis revealed that the rpoE2-chrR operon and these identified RpoE2 regulon member genes (SO_1987, SO_3348-SO_3349, SO_3374-SO_3386, and SO_4169-SO_4170) are coincidently absent in several Shewanella strains, including S. pealean ATCC 700345 [47], S. sediminis HAW-EB3 [48], S. piezotolerans WP3 [49], S. halifaxensis HAW-EB4 [50], S. violacea DSS12 [29,51], and S. benthica KT99 [52], which are deep-sea and/or psychrophilic strains [53].

\section{Discussion}

In this study the cellular functions of two RpoE-ECF sigma factors of $S$. oneidensis were investigated by comparative genomics, molecular genetics and physiological analyses. We have shown that RpoE is required for bacterial response to a series of stresses, including nutrient depletion (minimal medium), high salinity ( $3 \%$ sodium chloride), high and cold temperatures $\left(33^{\circ} \mathrm{C}\right.$ and $\left.4{ }^{\circ} \mathrm{C}\right)$, and oxidative stresses (hydrogen peroxide and paraquat) in the S. oneidensis MR-1 strain. On the other hand, RpoE2 is only involved in oxidative stress responses.

In $E$. coli and $P$. aeruginosa, the rpoE/alg $U$ gene is autoregulated because an $\mathrm{RpoE} / \mathrm{AlgU}$-dependent promoter is located upstream of this gene [23,24]. RpoE regulates a series of extracytoplasmic functions, including synthesis of envelope proteins, outer membrane protein (OMP) modification, cell envelope structure and cell division in E. coli [23]. The RpoE counterpart AlgU/T controls the production of a series of pathogenic factors, lipoproteins, and the extracellular polysaccharide alginate in $P$. aeruginosa which causes the mortality and morbidity of patients with cystic fibrosis [24-26]. RpoE is involved in biogenesis of envelope and integrity maintenance as previously demonstrated in mesophilic and psychrophilic bacteria [54]. Our results are consistent with previous microarray analysis data that the rpoE exhibited altered transcription under several stress conditions (summarized in the Additional file 1: Table S4). 

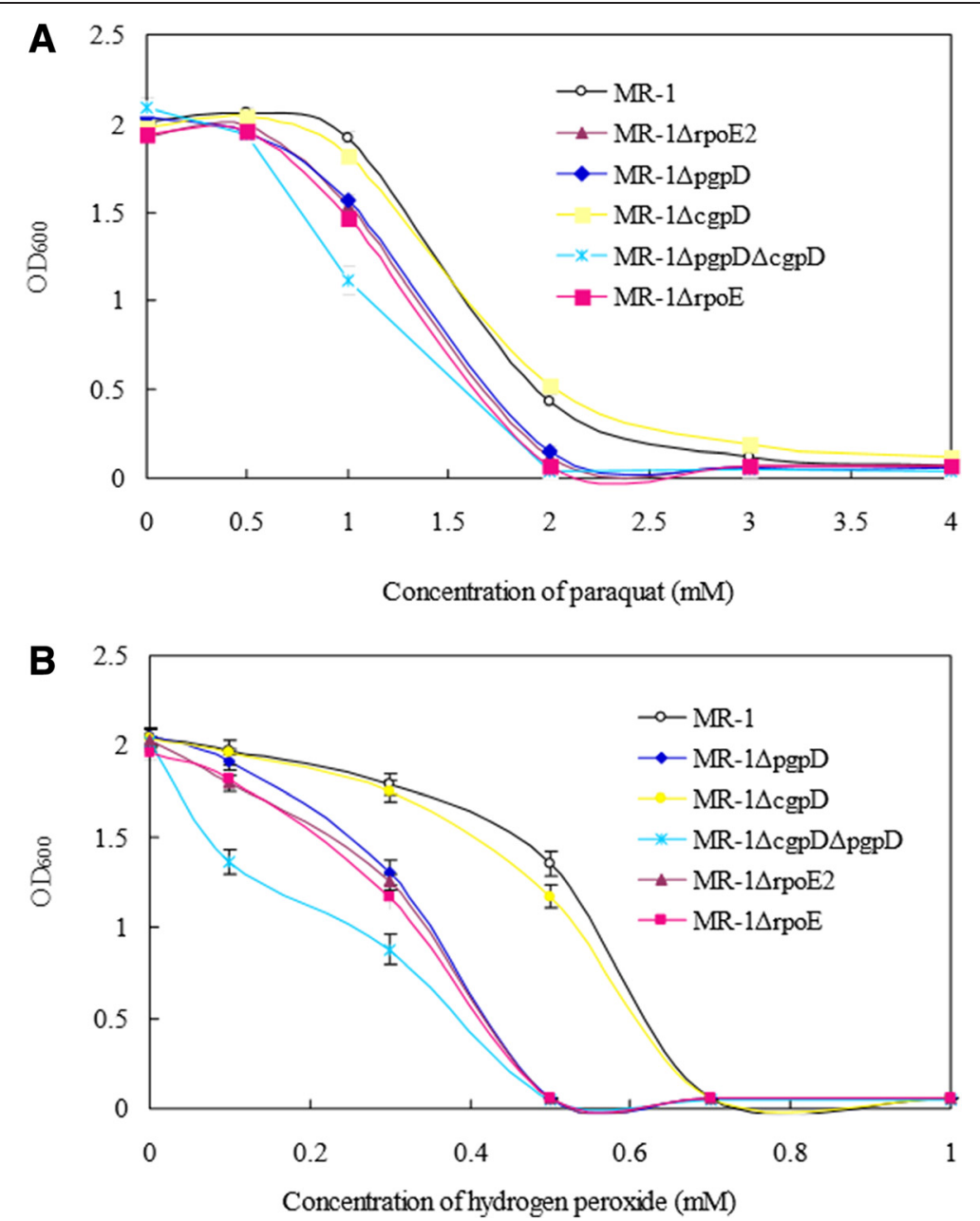

Figure 5 Effects of paraquat and hydrogen peroxide $\left(\mathrm{H}_{2} \mathrm{O}_{2}\right)$ on the bacterial growth of the Shewanella oneidensis strains. MR-1 wild type strain, the rpoE2, pgpD (SO_3349) and cgpD (SO_1563) in-frame deletion mutants and the pgpD-cgpD double mutant strains were grown in the LB broth containing A) $0,0.5,1,2,3$, and $4 \mathrm{mM}$ of paraquat or B) $0,0.1,0.3,0.5,0.7$ and $1 \mathrm{mM}$ of hydrogen peroxide and incubated at $28^{\circ} \mathrm{C}$ for $18 \mathrm{hrs}$.

The N-terminal domain of ChrR of R. sphaeroides is structurally similar to that of RseA of $E$. coli and defines a common cupin fold among anti- $\sigma$ factors $[44,45]$.

The Shewanella strains harbor a large number of $c$-type cytochrome genes for respiration. A total of 32 and $41 c$-type cytochrome genes are present in $S$. putrefaciens W3-18-1 and S. oneidensis MR-1, respectively [29]. These cytochromes and respiratory chains are a potential source of singlet oxygen $[44,45]$, which may account for the presence of the rpoE2-chrR pair and the periplasmic glutathione peroxidase gene $p g p D$ in most of the sequenced Shewanella strains. On the other hand, the rpoE2-chrR pair and the identified regulon members are coincidentally absent in the deep-sea/psychrophilic strains of Shewanella. The deep-sea water is characterized by a very low temperature, typically from $0^{\circ} \mathrm{C}$ to $3^{\circ} \mathrm{C}$, a high salinity of about $3.5 \%$, as well as low radiation. As described above, rpoE2 was not required for bacterial growth under high temperature, nutrient deficiency and particularly cold temperature and high salinity encountered in deep-sea environments. More importantly, the deletion of rpoE2 even enhanced the bacterial growth under salt stress condition (Figure $2 \mathrm{E}$ and Additional file 1: Figure S1). On the other hand, overexpression of rpoE2 affected bacterial growth under salt stress condition (Additional file 1: Figure S12). These results indicated a tradeoff between oxidative stress response and salt stress tolerance. The loss of these genes may represent a bacterial adaptation to deep-sea and cold environments of high salinity. It remains intriguing why RpoE2-mediated changes affect the bacterial growth under high salinity. The functions and regulation of other ECF os remain largely unknown in Shewanella [30]. The signaling mechanism for the activation and regulon of each $\sigma$ factor need to be experimentally investigated since their functions could not be completely predicted based on the existing knowledge from the closely related bacteria and comparative genomics analyses as shown by our results. 

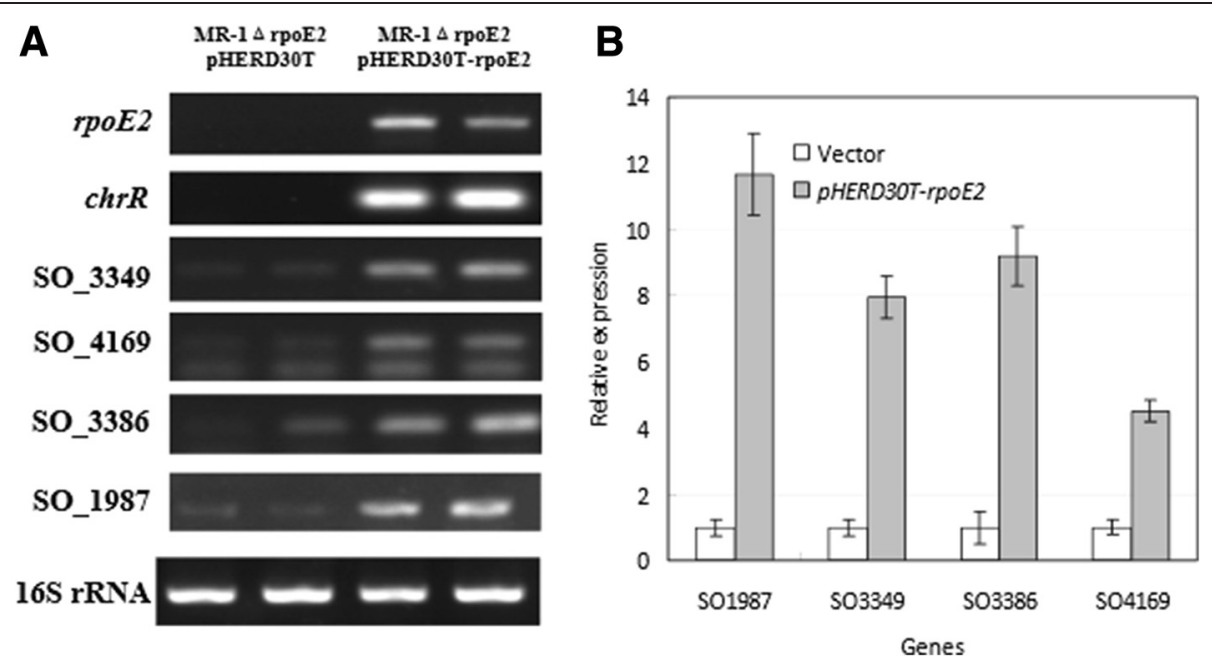

Figure 6 Induced transcription of the member genes of RpoE2-regulated operons in the rpoE2 null in-frame deletion mutant (MR-1 $\Delta$ rpoE2) carrying the plasmid-borne rpoE2 gene. The strain carrying PHERD30T empty vector was used as control and $0.01 \%$ (w/v) of L-arabinose was added to the bacterial cultures of both control (carrying pHERD30T vector) and treatment (carrying pHERD30T-rpoE2) during late exponential phase $\left(\mathrm{OD}_{600}>0.8\right)$. The cells were collected for RNA extract after 1 hour of induction. A) Transcription of the genes was examined by using semi-quantitative RT-PCR; 165 rRNA gene exp ression was analyzed and used as the loading control. B) Trace quantity plotting of figure 6A using 'Quantity One' software.The quantitative data represents three times of assays in duplicates.

\section{Conclusions}

Two of the ECF sigma factors, RpoE and RpoE2, regulate a series of extracytoplasmic functions in S. oneidensis MR-1. It is revealed that the RpoE-dependent $\operatorname{deg} Q$ gene is required for optimal growth under high temperature. The rpoE2 and RpoE2-dependent $p g p D$ gene are involved in oxidative stress responses. The glutathione peroxidase PgpD is secreted into the periplasm and plays a more important role in oxidative stress responses than the cytoplasmic homlog CgpD. But rpoE2 is not required for bacterial growth at low temperature and it even affected bacterial growth under salt stress, indicating that there is a tradeoff between the salt resistance and RpoE2-mediated oxidative stress responses.

\section{Methods}

Bacterial strains, plasmids, culture conditions and genome sequences

The bacterial strains and plasmid used in this study were listed in Additional file 1: Table S1. Bacterial strains were usually cultured in Lysogeny Broth (LB) (containing $10 \mathrm{~g}$ tryptone, $5 \mathrm{~g}$ yeast extract, and $5 \mathrm{~g}$ sodium chloride per litre) media/plates and the modified M1 minimum media (50 mM sodium lactate was used as a carbon source. when necessary, supplemented with 15 and $50 \mu \mathrm{g} / \mathrm{ml}$ of gentamycin and kanamycin, respectively) [32]. $S$. oneidensis MR-1 (ATCC 700550) was isolated from the sediment of Lake Oneida, New York [3] and usually incubated at $28^{\circ} \mathrm{C}$ in our laboratory. The whole genome
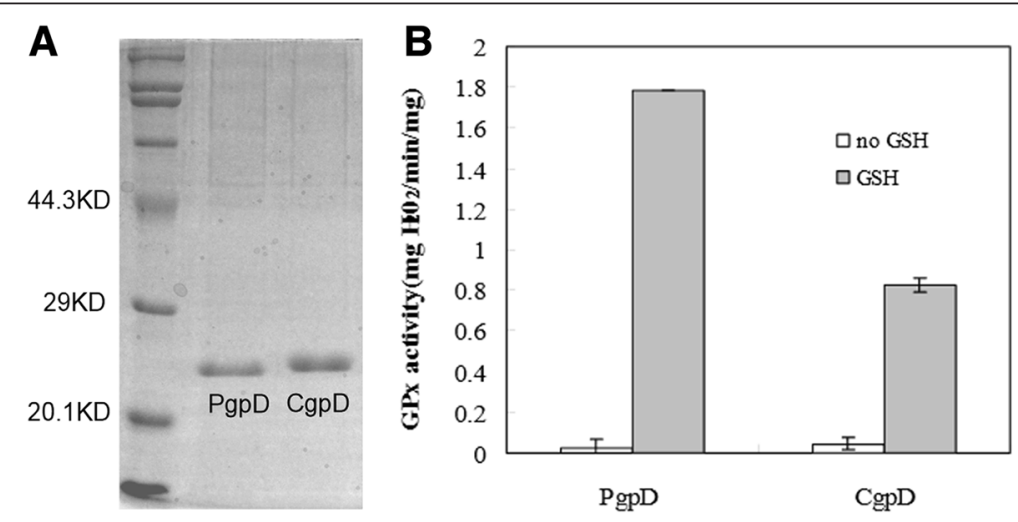

Figure 7 Glutathione (GSH) peroxidase (GPx) activity assays of PgpD and CgpD. A) Histidine-tagged CgpD and PgpD proteins were purified. B) Glutathione was added and the peroxidase activity is defined as the amount of hydrogen peroxide (mg) broken down in one minute (min) by one $\mathrm{mg}$ of purified enzymes $\left(\mathrm{mg} \mathrm{H}_{2} \mathrm{O}_{2} / \mathrm{min} / \mathrm{mg}\right)$ under the assay conditions described. 
sequences for around 30 strains of Shewanella are available at the NCBI microbial genome database. The genome of $S$. oneidensis MR-1 was sequenced and annotated by J. Craig Venter Institute [55] and other strains were analyzed by Joint Genome Institute and other institutions. The geographical origin of Shewanella strains and isolation site characteristics were summarized previously [1,2]. Pseudomonas aeruginosa PAO1 strain was obtained from ATCC (Manassas, VA, USA).

\section{Bioinformatics tools}

Polypeptide and nucleotide sequences of genes were retrieved from NCBI database by using BLAST searches. The orthologous relationships among the homologous genes from each bacterial genome are identified by using bidirectional BLASTP searches (best hits) and also based on synteny. The Clustal W package (http://ebi.ac.uk/clustalw) was used for polypeptide and nucleotide sequence alignments and phylogenetic footprinting analyses of promoters and Weblogo (http://weblogo.berkeley.edu) was applied to nucleotide sequence motif identification. The cellular localization of proteins in the cytoplasmic or periplasmic compartment was predicted using Signal P 4.1 server (http://cbs.dtu.dk/services/SignalP).

\section{Genetic manipulation and genetic complementation}

The two-step protocol of selection (gentamycin resistance $\left(\mathrm{Gm}^{\mathrm{R}}\right)$ for single cross-over) and counter-selection (sucrose sensitivity for double crossover) was applied for in-frame deletion of specific genes using the suicide vector pDS3.0 (R6K replicon, $s a c B, \mathrm{Gm}^{\mathrm{R}}$ )-based constructs with a fusion of upstream and downstream sequences as previously described [56]. The genes of S. oneidensis MR-1 was PCR amplified and cloned into the pHERD30T shuttle vector, which is suitable for cloning of toxic and tightly regulated genes like ECF sigma factor genes [57]. The resultant constructs and empty vector were transferred into the MR-1 wild type strain and mutants as well as the $P$. aeruginosa $\mathrm{PAO} 1$ via conjugation.

\section{RNA extraction, CDNA synthesis and RT-PCR analysis of gene transcription}

Total RNA was extracted by using RNAiso Plus (Takara, Dalian, China) or RNAprep pure Cell/Bacteria Kit (Tiangen Biotech, Beijing, China) and RNA was further purified using DNase I treatment. The integrity of RNA was evaluated by agarose $(0.8 \%)$ gel electrophoresis. The RNA concentration and purity was measured on a spectrophotometer (Nanodrop Technologies, Wilmington, DE, USA). To prepare cDNA, $2 \mu \mathrm{g}$ of total RNA was reversely transcribed using PrimeScript ${ }^{\circ}$ RT reagent Kit with gDNA Eraser (Takara, Dalian, China) and TIANscript RT Kit (TIANGEN BIOTECH (Beijing) CO., LTD.) according to the manufacturer's protocol. The PCR thermal cycles were:
5 min at $95^{\circ} \mathrm{C}$ for cDNA denaturation, followed by $27-30$ cycles of $30 \mathrm{~s}$ at $95^{\circ} \mathrm{C}, 30 \mathrm{~s}$ at $51-60^{\circ} \mathrm{C}$ and $30 \mathrm{~s}$ at $72^{\circ} \mathrm{C}$. A final elongation step was performed for $10 \mathrm{~min}$ at $72^{\circ} \mathrm{C}$. RT-PCR products were electrophoresed in a $0.8 \%$ agarose gel containing ethidium bromide and visualized by ultraviolet light and Bio-Rad Image software. The data presented are relative mRNA levels normalized against $16 \mathrm{~S}$ rRNA transcript levels, and the value of the control was set to 1 . All the experiments described were performed in triplicates or repeated three times in duplicates to obtain means and standard deviation (SD). The PCR products were also sequenced to confirm amplification of target genes. The primers used were listed in the supplemental materials (Additional file 1: Table S2).

\section{Determination of transcription start site}

Terminal deoxynucleotidyl transferase (TdT, Takara) was used to catalyze the incorporation of single deoxynucleotides (dATPs) into the 3'-OH terminus of cDNA to make the dA-tailed cDNA according to the producer's protocol. Touchdown and nest PCR was used to amplify the dA-tailed cDNA by using an oligdT (5'-gccagtcTTT TTTTTTTTTTTTTT-3') primer and a specific primer [58]. The PCR product was cloned into pMD18-T vector (Takara, Dalian, China) for sequencing.

\section{Hydrogen peroxide and paraquat sensitivity assay}

To test the bacterial resistance to hydrogen peroxide $(0,0.1,0.3,0.5,0.7$ and $1 \mathrm{mM})$ and paraquat $(0,0.5$, $1,2,3$, and $4 \mathrm{mM}$ ) (1,1-dimethyl-4,4'-bipyridylium dichloride, a powerful propagator of superoxide radicals, Tokyo Chemical Industry Co., Tokyo, Japan) cells were grown overnight in LB broth containing different levels of each chemical and growth was monitored by measure optical density at $600 \mathrm{~nm}$ as previously described [59].

\section{Expression, extraction and activity assays of hydrogen peroxidases}

The glutathione hydrogen peroxidase (GPx) genes $p g p D$ (lacking the $\mathrm{N}$-terminal sequence encoding the signal peptide) and $c g p D$ were cloned into the $\mathrm{pET} 28$ a vector and the overproducing constructs were transferred into E. coli DE3/BL21 cells. The DE3 strains were grown in LB medium (supplemented with $100 \mu \mathrm{g} / \mathrm{L}$ of ampicillin) at $37^{\circ} \mathrm{C}$ to an $\mathrm{OD}_{600}$ of approximately 0.6 and the gene expression was induced by addition of IPTG $(0.01 \%$, w/v) at $16^{\circ} \mathrm{C}$ for 24 hours. The harvested E. coli cells were homogenized by applying high pressures (JN-02C low temperature ultra-high pressure continuous flow cell disrupter, Juneng Biol. \& Technol. Co., Guangzhou, China) and the His-tagged recombinant proteins were purified by using Ni-NTA Sepharose (GE Healthcare, Waukesha, Wisconsin, USA) affinity chromatography according to the supplier's protocol. The activity of hydrogen peroxidases is 
assayed by a widely used protocol with some modifications [60]. $3 \mathrm{ml}$ of the enzyme elute from Ni-NTA Sepharose was mixed with $3 \mathrm{ml}$ of phosphate buffer containing $0.1 \mathrm{M}$ hydrogen peroxide and $0.1 \mathrm{M}$ glutathione (GSH). The reaction was stopped by adding $3 \mathrm{ml}$ of $10 \%(\mathrm{v} / \mathrm{v})$ sulfuric acid and the residual hydrogen peroxide was titrated against $0.1 \mathrm{M}$ permanganate $\left(\mathrm{KMnO}_{4}\right)$ solution until a faint purple color persisted for at least 30 seconds. The enzyme concentrations were measured by using a total protein assay kit (Jiancheng Biotech., Nanjing, China). The same amounts of boiling-denatured enzyme solutions were used as control.

\section{Alkaline phosphatase A-fusion assay}

To determine the protein cellular location, the 5 '-nucleotide sequence, encoding the amino-terminal signal peptide (SP), of the $p g p D$ gene was translationally fused with $E$. coli phoA gene with deletion of the sequence encoding the $\mathrm{N}$-terminal signal sequence. This $p g p D-p h o A$ fusion and pho $A$ were cloned into pUCP20T vector for alkaline phosphatase A-fusion assay [46], and the transformants of DH5 $\alpha$ were plated on the LB plate containing $40 \mu \mathrm{g} / \mathrm{ml}$ of BCIP (5-Bromo-4-chloro-3-indolyl phosphate p-toluidine, Amresco, Solon, OH, USA) and $100 \mu \mathrm{g} / \mathrm{ml}$ of ampicillin. The construct pUCP20-phoA(wt) expressing full-length PhoA was used as positive control and the pUCP20-phoA (NSP) expressing the truncated PhoA without N-terminal signal leader sequence as negative control.

\section{Additional file}

Additional file 1: Supplemental Tables S1-S5 and Figures S1-S12 associated with this manuscript.

\section{Competing interests}

The authors declare that they have no competing interests.

\section{Authors' contributions}

JD and DQ generated the constructs and strains used. JD, HW, CT and DQ generated and analyzed the results. DQ and JZ designed the study. JD, HD and DQ drafted the manuscript. All authors read and approved the final manuscript.

\section{Acknowledgements}

This work was supported by the Chinese Academy of Science Grant Y15103-1-401 and One-Hundred Scholar Award to D.Q. and the DOE grant DE-FG02-07ER64383 to J. Z.

\footnotetext{
Author details

${ }^{1}$ Institute of Hydrobiology, Chinese Academy of Sciences, 7 South Donghu Road, Wuchang District, Wuhan 430072, China. ${ }^{2}$ University of Chinese Academy of Sciences, Beijing 100049, China. ${ }^{3}$ School of Life Sciences and Technology, Hubei University of Engineering, 272 Jiaotong Avenue, Xiaogan 432000, China. ${ }^{4}$ Division of Infectious Diseases and International Health, Department of Medicine, University of Virginia, Charlottesville, VA 22908, USA. ${ }^{5}$ Institute for Environmental Genomics and Department of Botany and Microbiology, The University of Oklahoma, Stephenson Research and Technology Center, 101 David L. Boren Blvd, Norman OK 73019, USA.
}

Received: 8 July 2014 Accepted: 20 January 2015

Published online: 18 February 2015

\section{References}

1. Hau HH, Gralnick JA. Ecology and biotechnology of the genus Shewanella. Annu Rev Microbiol. 2007;61:237-58.

2. Fredrickson JK, Romine MF, Beliaev AS, Auchtung JM, Driscoll ME, Gardner TS, et al. Towards environmental systems biology of Shewanella. Nat Rev Microbiol. 2008;6(8):592-603.

3. Myers $\mathrm{CR}$, Nealson $\mathrm{KH}$. Bacterial manganese reduction and growth with manganese oxide as the sole electron-acceptor. Science. 1988;240 (4857):1319-21.

4. Gorby YA, Yanina S, McLean JS, Rosso KM, Moyles D, Dohnalkova A, et al. Electrically conductive bacterial nanowires produced by Shewanella oneidensis strain MR-1 and other microorganisms. Proc Natl Acad Sci U S A. 2006;103(30):11358-63.

5. Gruber TM, Gross CA. Multiple sigma subunits and the partitioning of bacterial transcription space. Annu Rev Microbiol. 2003;57:441-66.

6. Hughes KT, Mathee K. The anti-sigma factors. Annu Rev Microbiol. 1998;52:231-86.

7. Helmann JD. The extracytoplasmic function (ECF) sigma factors. Adv Microb Physiol. 2002;46:47-110.

8. Potvin E, Sanschagrin F, Levesque RC. Sigma factors in Pseudomonas aeruginosa. Fems Microbiol Rev. 2008;32(1):38-55

9. Staron A, Sofia HJ, Dietrich S, Ulrich LE, Liesegang H, Mascher T. The third pillar of bacterial signal transduction: classification of the extracytoplasmic function (ECF) sigma factor protein family. Mol Microbiol. 2009;74(3):557-81.

10. Alba BM, Leeds JA, Onufryk C, Lu CZ, Gross CA. DegS and YaeL participate sequentially in the cleavage of RseA to activate the sigma(E)-dependent extracytoplasmic stress response. Gene Dev. 2002;16(16):2156-68.

11. Alba BM, Zhong HJ, Pelayo JC, Gross CA. degS (hhoB) is an essential Escherichia coli gene whose indispensable function is to provide sigma(E) activity. Mol Microbiol. 2001;40(6):1323-33.

12. Walsh NP, Alba BM, Bose B, Gross CA, Sauer RT. OMP peptide signals initiate the envelope-stress response by activating DegS protease via relief of inhibition mediated by its PDZ domain. Cell. 2003;113(1):61-71.

13. Grigorova IL, Chaba R, Zhong HJ, Alba BM, Rhodius V, Herman C, et al. Fine-tuning of the Escherichia coli sigma(E) envelope stress response relies on multiple mechanisms to inhibit signal-independent proteolysis of the transmembrane anti-sigma factor. RseA. Gene Dev. 2004;18(21):2686-97.

14. Kanehara K, Ito K, Akiyama Y. YaeL proteolysis of RseA is controlled by the PDZ domain of YaeL and a Gln-rich region of RseA. Embo J. 2003;22(23):6389-98.

15. Murata M, Fujimoto H, Nishimura K, Charoensuk K, Nagamitsu H, Raina S, et al. Molecular strategy for survival at a critical high temperature in Eschierichia coli. Plos One. 2011;6(6):e20063.

16. Noor R, Murata M, Nagamitsu H, Klein G, Raina S, Yamada M. Dissection of sigma(E)-dependent cell lysis in Escherichia coli: roles of RpoE regulators RseA, RseB and periplasmic folding catalyst PpiD. Genes Cells. 2009;14(7):885-99.

17. Murata M, Noor R, Nagamitsu H, Tanaka S, Yamada M. Novel pathway directed by $\sigma^{\mathrm{E}}$ to cause cell lysis in Escherichia coli. Genes Cells. 2012;17(3):234-47.

18. Qiu DR, Eisinger VM, Rowen DW, Yu HWD. Regulated proteolysis controls mucoid conversion in Pseudomonas aeruginosa. Proc Natl Acad Sci U S A. 2007;104(19):8107-12.

19. Qiu DR, Esinger VM, Head NE, Pier GB, Yu HD. ClpXP proteases positively regulate alginate overexpression and mucoid conversion in Pseudomonas aeruginosa. Microbiol-Sgm. 2008;154:2119-30.

20. Wood LF, Ohman DE. Use of cell wall stress to characterize sigma(22) (AlgT/U) activation by regulated proteolysis and its regulon in Pseudomonas aeruginosa. Mol Microbiol. 2009;72(1):183-201.

21. Cezairliyan BO, Sauer RT. Control of Pseudomonas aeruginosa AlgW protease cleavage of MucA by peptide signals and MucB. Mol Microbiol. 2009;72(2):368-79.

22. Damron FH, Goldberg JB. Proteolytic regulation of alginate overproduction in Pseudomonas aeruginosa. Mol Microbiol. 2012;84(4):595-607.

23. Rhodius VA, Suh WC, Nonaka G, West J, Gross CA. Conserved and variable functions of the sigmaE stress response in related genomes. PLoS Biol. 2006;4(1):e2. 
24. Firoved AM, Deretic V. Microarray analysis of global gene expression in mucoid Pseudomonas aeruginosa. J Bacteriol. 2003;185(3):1071-81.

25. Martin DW, Holloway BW, Deretic V. Characterization of a locus determining the mucoid status of Pseudomonas aeruginosa - AlgU shows sequence similarities with a Bacillus sigma-factor. J Bacteriol. 1993;175(4):1153-64.

26. Govan JRW, Deretic V. Microbial pathogenesis in cystic fibrosis: mucoid Pseudomonas aeruginosa and Burkholderia cepacia. Microbiol Rev. 1996;60(3):539-74.

27. Gao HC, Wang Y, Liu XD, Yan TF, Wu LY, Alm E, et al. Global transcriptome analysis of the heat shock response of Shewanella oneidensis. J Bacteriol. 2004;186(22):7796-803.

28. Kabir MS, Yamashita D, Koyama S, Oshima T, Kurokawa K, Maeda M, et al. Cell lysis directed by sigmaE in early stationary phase and effect of induction of the rpoE gene on global gene expression in Escherichia coli. Microbiology. 2005;151:2721-35.

29. Kawano H, Abe F, Nakasone K, Kato C, Yoshida Y, Usami R, et al. Cloning and characterization of the rpoE gene encoding an RNA polymerase sigmaE factor from the deep-sea piezophilic Shewanella violacea strain DSS12. DNA Seq. 2005;16(1):69-74.

30. Kawano H, Takahashi H, Abe F, Kato C, Horikoshi K. Identification and characterization of two alternative sigma factors of RNA polymerase in the deep-sea piezophilic bacterium Shewanella violacea, Strain DSS12. Biosci Biotech Bioch. 2009;73(1):200-2

31. Liu YQ, Gao WM, Wang Y, Wu LY, Liu XD, Yan TF, et al. Transcriptome analysis of Shewanella oneidensis MR-1 in response to elevated salt conditions. J Bacteriol. 2005;187(7):2501-7.

32. Beliaev AS, Klingeman DM, Klappenbach JA, Wu L, Romine MF, Tiedje JA, et al. Global transcriptome analysis of Shewanella oneidensis MR-1 exposed to different terminal electron acceptors. J Bacteriol. 2005;187(20):7138-45.

33. Qiu XY, Sundin GW, Wu LY, Zhou JZ, Tiedje JM. Comparative analysis of differentially expressed genes in Shewanella oneidensis MR-1 following exposure to UVC, UVB, and UVA radiation. J Bacteriol. 2005;187(10):3556-64.

34. Leaphart AB, Thompson DK, Huang K, Alm E, Wan XF, Arkin A, et al. Transcriptome profiling of Shewanella oneidensis gene expression following exposure to acidic and alkaline pH. J Bacteriol. 2006;188(4):1633-42.

35. Bencheikh-Latmani R, Williams SM, Haucke L, Criddle CS, Wu LY, Zhou JZ, et al. Global transcriptional profiling of Shewanella oneidensis MR-1 during $\mathrm{Cr}(\mathrm{VI})$ and $\mathrm{U}(\mathrm{VI})$ reduction. Appl Environ Microb. 2005;71(11):7453-60.

36. Brown SD, Martin M, Deshpande S, Seal S, Huang K, Alm E, et al. Cellular response of Shewanella oneidensis to strontium stress. Appl Environ Microb. 2006;72(1):890-900.

37. Chourey K, Thompson MR, Morrell-Falvey J, VerBerkmoes NC, Brown SD, Shah $\mathrm{M}$, et al. Global molecular and morphological effects of 24-hour chromium(VI) exposure on Shewanella oneidensis MR-1. Appl Environ Microb. 2006;72(9):6331-44.

38. Qiu DR, Wei HH, Tu QC, Yang YF, Xie M, Chen JR, et al. Combined genomics and experimental analyses of respiratory characteristics of Shewanella putrefaciens W3-18-1. Appl Environ Microb. 2013;79(17):5250-7.

39. Anthony JR, Warczak KL, Donohue TJ. A transcriptional response to singlet oxygen, a toxic byproduct of photosynthesis. Proc Natl Acad Sci U S A. 2005;102(18):6502-7.

40. Poirel L, Heritier C, Nordmann P. Chromosome-encoded ambler class D beta-lactamase of Shewanella oneidensis as a progenitor of carbapenem-hydrolyzing oxacillinase. Antimicrob Agents Ch. 2004:48(1):348-51.

41. Krojer T, Garrido-Franco M, Huber R, Ehrmann M, Clausen T. Crystal structure of DegP $(\mathrm{Htr} A)$ reveals a new protease-chaperone machine. Nature. 2002:416(6879):455-9.

42. Wootton JC, Drummond MH. The Q-Linker - a class of interdomain sequences found in bacterial multidomain regulatory proteins. Protein Eng. 1989;2(7):535-43.

43. Dartigalongue C, Missiakas D, Raina S. Characterization of the Escherichia coli sigma(E) regulon. J Biol Chem. 2001;276(24):20866-75.

44. Dufour YS, Landick R, Donohue TJ. Organization and evolution of the biological response to singlet oxygen stress. J Mol Biol. 2008;383(3):713-30.

45. Campbell EA, Greenwell R, Anthony JR, Wang S, Lim L, Das K, et al. A conserved structural module regulates transcriptional responses to diverse stress signals in bacteria. Mol Cell. 2007;27(5):793-805.

46. Hoffman CS, Wright A. Fusions of secreted proteins to alkaline-phosphatase an approach for studying protein secretion. Proc Natl Acad Sci U S A. 1985;82(15):5107-11.
47. Leonardo MR, Moser DP, Barbieri E, Brantner CA, MacGregor BJ, Paster BJ, et al. Shewanella pealeana sp nov., a member of the microbial community associated with the accessory nidamental gland of the squid Loligo pealei. Int J Syst Bacteriol. 1999:49:1341-51.

48. Zhao JS, Manno D, Beaulieu C, Paquet L, Hawari J. Shewanella sediminis sp. nov., a novel $\mathrm{Na}^{+}$-requiring and hexahydro-1,3,5-trinitro-1,3,5-triazine-degrading bacterium from marine sediment. Int J Syst Evol Microbiol. 2005;55(Pt 4):1511-20.

49. Xiao X, Wang P, Zeng X, Bartlett DH, Wang F. Shewanella psychrophila sp. nov. and Shewanella piezotolerans sp. nov., isolated from west Pacific deep-sea sediment. Int J Syst Evol Microbiol. 2007;57(Pt 1):60-5.

50. Zhao JS, Manno D, Leggiadro C, O'Neil D, Hawari J. Shewanella halifaxensis sp. nov., a novel obligately respiratory and denitrifying psychrophile. Int J Syst Evol Microbiol. 2006;56(Pt 1):205-12.

51. Aono E, Baba T, Ara T, Nishi T, Nakamichi T, Inamoto E, et al. Complete genome sequence and comparative analysis of Shewanella violacea, a psychrophilic and piezophilic bacterium from deep sea floor sediments. Mol Biosyst. 2010;6(7):1216-26.

52. Lauro FM, Chastain RA, Ferriera S, Johnson J, Yayanos AA, Bartlett DH. Draft genome sequence of the deep-sea bacterium Shewanella benthica Strain KT99. Genome Announc. 2013;1(3):e00210-13.

53. Karpinets TV, Obraztsova AY, Wang YB, Schmoyer DD, Kora GH, Park BH, et al. Conserved synteny at the protein family level reveals genes underlying Shewanella species' cold tolerance and predicts their novel phenotypes. Funct Integr Genomic. 2010;10(1):97-110.

54. Chi E, Bartlett DH. An RpoE-like locus controls outer-membrane protein-synthesis and growth at cold temperatures and high-pressures in the deep-sea bacterium Photobacterium Sp Strain Ss9. Mol Microbiol. 1995;17(4):713-26.

55. Heidelberg JF, Paulsen IT, Nelson KE, Gaidos EJ, Nelson WC, Read TD, et al. Genome sequence of the dissimilatory metal ion-reducing bacterium Shewanella oneidensis. Nat Biotechnol. 2002;20(11):1118-23.

56. Wan XF, VerBerkmoes NC, McCue LA, Stanek D, Connelly H, Hauser LJ, et al. Transcriptomic and proteomic characterization of the fur modulon in the metal-reducing bacterium Shewanella oneidensis. J Bacteriol. 2004;186(24):8385-400.

57. Qiu DR, Damron FH, Mima T, Schweizer HP, Yu HD. P-BAD-based shuttle vectors for functional analysis of toxic and highly regulated genes in Pseudomonas and Burkholderia spp. and Other Bacteria. Appl Environ Microb. 2008;74(23):7422-6.

58. Mendoza-Vargas A, Olvera L, Olvera M, Grande R, Vega-Alvarado L, Taboada B, et al. Genome-wide identification of transcription start sites, promoters and transcription factor binding sites in E. coli. Plos One. 2009:4(10):e7526

59. Won SH, Lee BH, Lee HS, Jo J. An Ochrobactrum anthropi gene conferring paraquat resistance to the heterologous host Escherichia coli. Biochem Bioph Res Co. 2001;285(4):885-90.

60. Kar M, Mishra D. Catalase, peroxidase, and polyphenoloxidase activities during rice leaf senescence. Plant Physiol. 1976;57(2):315-9.

\section{Submit your next manuscript to BioMed Central and take full advantage of:}

- Convenient online submission

- Thorough peer review

- No space constraints or color figure charges

- Immediate publication on acceptance

- Inclusion in PubMed, CAS, Scopus and Google Scholar

- Research which is freely available for redistribution 